\title{
Neuroprotective strategies against calpain-mediated neurodegeneration
}

This article was published in the following Dove Press journal:

Neuropsychiatric Disease and Treatment

5 February 2015

Number of times this article has been viewed

Aysegul Yildiz-Unal'

Sirin Korulu²

Arzu Karabay ${ }^{3}$

'Department of Molecular Biology and Genetics, Faculty of Science, Muğla Sıtkı Koçman University, Kötekli, Muğla, Turkey; ${ }^{2}$ Department of Molecular Biology and Genetics, Istanbul Arel University, Istanbul Turkey; ${ }^{3}$ Department of Molecular Biology and Genetics, Faculty of Science and Letters, Istanbul Technical University, Maslak, Istanbul, Turkey
Correspondence: Aysegul Yildiz-Unal Department of Molecular Biology and Genetics, Faculty of Science, Muğla Sıtkı Koçman University, Kötekli, Muğla, 48000, Turkey

Tel +9025221 I 5412

Fax +90 252 2II |472

Email aysegulunal@mu.edu.tr
Abstract: Calpains are calcium-dependent proteolytic enzymes that have deleterious effects on neurons upon their pathological over-activation. According to the results of numerous studies to date, there is no doubt that abnormal calpain activation triggers activation and progression of apoptotic processes in neurodegeneration, leading to neuronal death. Thus, it is very crucial to unravel all the aspects of calpain-mediated neurodegeneration in order to protect neurons through eliminating or at least minimizing its lethal effects. Protecting neurons against calpain-activated apoptosis basically requires developing effective, reliable, and most importantly, therapeutically applicable approaches to succeed. From this aspect, the most significant studies focusing on preventing calpain-mediated neurodegeneration include blocking the $N$-methyl-D-aspartate (NMDA)-type glutamate receptor activities, which are closely related to calpain activation; directly inhibiting calpain itself via intrinsic or synthetic calpain inhibitors, or inhibiting its downstream processes; and utilizing the neuroprotectant steroid hormone estrogen and its receptors. In this review, the most remarkable neuroprotective strategies for calpain-mediated neurodegeneration are categorized and summarized with respect to their advantages and disadvantages over one another, in terms of their efficiency and applicability as a therapeutic regimen in the treatment of neurodegenerative diseases.

Keywords: calpain, neurodegeneration, neuroprotection, calpain inhibitors, NMDAR, Speedy/ RINGO

\section{The role of pathological calpain activation in neurodegenerative diseases}

The term "neurodegeneration" defines the progressive loss of function and structure of neurons that finally ends up with neuronal cell death. Since neurodegeneration is a multifactorial and hence, a very complicated process, its exact mechanism still remains unknown, and its origin of onset is a type of "chicken and egg" situation. Despite its multifaceted nature, it is known that over-activation of cysteine-protease calpains as a result of destabilization of calcium homeostasis is one of the main causative factors for neurodegeneration. There are number of neurodegenerative conditions such as amyloid beta $(\mathrm{A} \beta)$ aggregation, metabolic alterations, and oxidative stress, which all cause destabilization of calcium homeostasis, resulting in the elevation of intracellular calcium levels, which in turn finally leads to pathological activation of calcium-sensitive cysteine-protease, calpain (Figure 1). Calpain is known to be one of the most effective proteins of which deregulation leads neurons into apoptosis through different pathways. ${ }^{1-4}$

Calpains are heterodimeric proteins that are composed of a large $80 \mathrm{kDa}$ catalytic subunit and a small $30 \mathrm{kDa}$ regulatory subunit. The small $30 \mathrm{kDa}$ subunit contains two domains: V and VI. Domain V is the terminal domain and possesses glycine residues, while domain VI has five helix-loop-helix structural EF-hand calcium-binding motifs. ${ }^{5}$ 


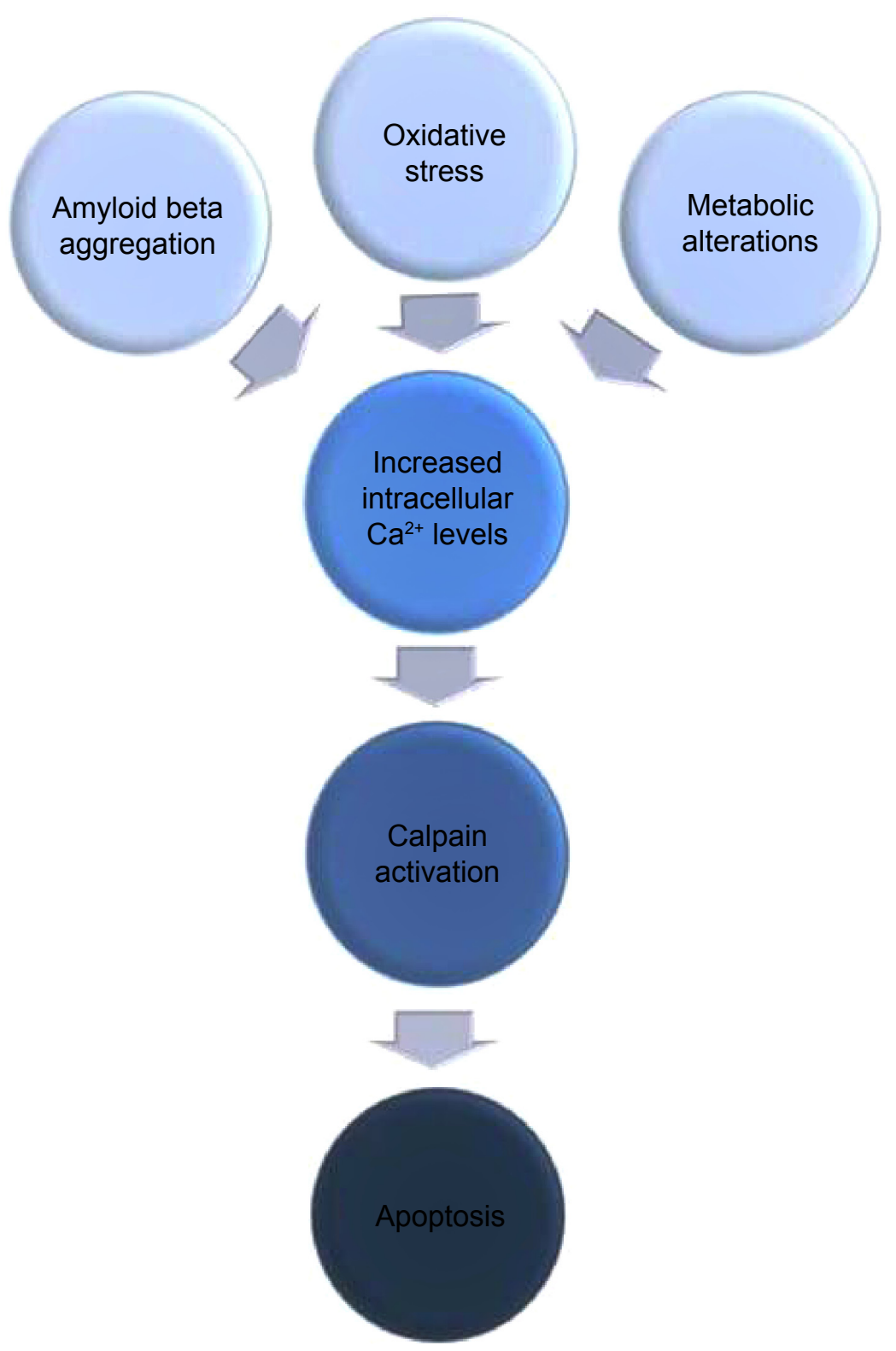

Figure I Schematic illustration of calpain-mediated apoptotic progression of neurons as a result of different neurodegenerative stimuli.

The large $80 \mathrm{kDa}$ subunit is composed of four domains: I, II, III, and IV. Domain I is the autolytic cleavage site, and domain II has the cysteine protease activity and interacts with the substrates. Domain III's function is not known. Domain IV contains E-helix-loop-F-helix motifs (EF hands) on which are calcium binding sites. ${ }^{6}$

Calpain is localized in the cytosol in its inactive form in the absence of calcium. Upon an increase in intracellular calcium level, calpain is translocated to the membrane, and there, it becomes activated with calcium and phospholipids. Calpain activation occurs mainly in two stages. In inactive form, two subdomains of protease domain II (IIa and IIb) are separated by structural constraints. In the first stage of activation, this structural constraint is released in the presence of calcium ions, which is a necessity to activate the calpain and to form the active catalytic site. ${ }^{7}$ Calcium binds to EF-hand motifs in domains III, IV, and VI, and this binding separates domain II from domain III. Therefore, a $30 \mathrm{kDa}$ subunit dissociates from an $80 \mathrm{kDa}$ subunit at the end of the first stage. At the second stage, an active site on the protease domain II is rearranged to be able to interact with its substrates. ${ }^{8}$

Although calpain activity is strictly regulated, mainly by calcium, there is also an endogenous inhibitor of calpain, namely calpastatin, which regulates the activity of calpain. ${ }^{9}$ Calpastatin reversibly binds to the active site and inhibits calpain only in the presence of calcium ions. ${ }^{10}$ 
Activated calpain has a number of substrates such as growth factor receptors, cytoskeletal proteins, microtubuleassociated proteins, and mitochondria in order to fulfill crucial roles in different cellular mechanisms such as progression of cell cycle, ${ }^{11}$ differentiation, apoptosis, longterm potentiation (LTP), synaptic plasticity, and central nervous system (CNS) development in neurons. ${ }^{12,13}$ LTP is a calcium-dependent regulator of memory development that results, in part, from remodeling of dendritic spines. ${ }^{14}$ Activation of $N$-methyl-D-aspartate (NMDA) receptors, which results in the activation of calpains and proteolysis of structural proteins such as spectrin, ${ }^{15}$ plays a crucial role in the induction of LTP. ${ }^{14}$ Different studies also showed that calpain inhibition caused rapid axon retraction, attributing a role for calpain in axon maturation and maintenance during CNS development. ${ }^{16,17}$

Recent studies have indicated that calpains play significant roles in the apoptotic processes in the case of neurodegeneration. ${ }^{1,18,19}$ Deregulation of calcium homeostasis results in calcium overload, and subsequent calpain overactivation forces neurons to ultimately undergo apoptosis for the reason that members of apoptotic machinery, such as B-cell lymphoma 2 (Bcl-2) family members of cell death regulators and nuclear transcription factors such as p53 and caspases are themselves direct substrates of calpains..$^{20}$

Several members of the Bcl-2 family proteins that regulate programmed cell death ${ }^{21}$ are processed by calpains. ${ }^{22}$ Putcha et $\mathrm{al}^{23}$ showed that Bax (a Bcl-2 family member) translocation from cytosol to mitochondria is a key event in neuronal apoptosis in the trophic factor-deprived sympathetic neuron model. The role of calpain in this process is to cleave Bax into an 18-kDa pro-apoptotic fragment that leads to cytochrome c release and subsequently, apoptosis. ${ }^{24}$

In addition to this, Sedarous et a ${ }^{16}$ showed that inhibiting calpain by pharmacological calpain inhibitors (MDL-28170 and PD 150606) significantly reduced p53 induction and subsequent cytochrome c release, and caspase- 3 increase in embryonic cortical neurons, indicating that calpain is a key mediator of p53 induction and caspase-dependent apoptosis.

In addition, experimental data suggest some neuroprotective effects for inhibitors of calpain and caspase in neurons. ${ }^{6,25}$ An increased body of evidence indicates an interaction between calpain and caspase proteolytic systems. Calpain can lead to activation of caspase-3 by cleaving pro-caspase-3. Caspases can also play a role in the degradation process of the specific endogenous calpain inhibitor, calpastatin, accelerating calpain activation. Moreover, in an experiment using ultraviolet radiation to trigger apoptosis, it has been shown that calpain activity is required for caspase- 3 activation. ${ }^{25,26}$ These findings obviously indicate that both calpain and caspase proteolytic systems are involved in the progression of neuronal death..$^{27,28}$

Since pathological calpain activation is one of the most important neurodegenerative factors causing activation of apoptotic machinery, it is crucial to develop effective and reliable approaches to prevent calpain-mediated apoptosis in degenerating neurons. An increasing number of studies demonstrated that there are many kinds of different stimuli that trigger pathological calpain activation. ${ }^{29-31}$ Thus, depending on the type of stimuli, there are numerous strategies developed by distinct research groups to inhibit apoptotic effects of calpain over-activity. Although using calpain inhibitors is the most frequently applied strategy for the blockade of calpain-mediated apoptosis in neurodegeneration, considering its disadvantages, some other neuroprotective strategies are also being utilized. The most frequently used methods for this reason are over-expression or inhibition of certain proteins, especially those are involved in glutamate receptor signaling or using receptor antagonists, testing certain hormones and their receptors for their neuroprotective activities or designing competitive peptides to inhibit calpain's enzymatic activity are also utilized.

\section{Neuroprotection through direct inhibition of glutamate receptor activity}

Glutamate is an important neurotransmitter of the CNS that functions in many physiological cellular events through activating glutamate receptors. ${ }^{32,33}$ On the other hand, glutamate can be toxic for neurons in the case of excessive or prolonged exposure, which is known as glutamate neurotoxicity. ${ }^{34,35}$ Glutamate neurotoxicity is known to be a major factor in a number of chronic neurodegenerative disorders such as amyotrophic lateral sclerosis and Alzheimer's disease. ${ }^{36}$

It has been shown that abnormal $\mathrm{Ca}^{2+}$ influx through glutamate receptors is a part of glutamate neurotoxicity, which results in activation of certain enzymes such as calpain, leading to cleavage and degradation of proteins, membranes, and nucleic acids. ${ }^{37}$ Although the underlying mechanisms of glutamate neurotoxicity are not completely known, the NMDA receptor (NMDAR)-mediated $\mathrm{Ca}^{2+}$ overload and subsequent calpain activation have been indicated as strong candidates. ${ }^{38,39}$

There is a growing body of evidence indicating involvement of NMDARs in calpain-mediated neuronal injury and 
neuronal death. In a study investigating the role of calpain in glutamate-induced retinal neuron injury, glutamate treatment has been shown to induce apoptosis by elevating $\mathrm{Ca}^{2+}$ influx and protein levels of calpain 2 and calpain-specific alpha-spectrin breakdown products (SBDPs). ${ }^{40}$ Together with calpain induction, an increase in cyclin-dependent kinase 5 (cdk5) and its co-activator p35 protein levels have been determined. Under normal conditions, p35 is the partner for cdk5, which is a non-mitotic neuron-specific kinase, and a cdk5/p35 complex is formed in important cellular events such as neuronal development and maturation. ${ }^{41-43}$ However, in the case of neuronal calpain over-activation, calpain cleaves p35 into p25 and p10 fractions, as p35 is a substrate of calpain. p25 has binding ability to cdk5 to form a cdk5/ p25 complex. However, p25 causes prolonged activation and mislocalization of cdk5. ${ }^{44}$ Owing to prolonged activation of cdk5 by p25, the calpain/p35-p25/cdk5/NMDAR signaling pathway is implicated in playing a role in neurological disorders by phosphorylation of NMDAR subunit NR2A at the Ser1232 site (p-NR2AS1232), induction of the functional NMDARs'expression in the cell membrane, and subsequent glutamate-induced increase of $\mathrm{Ca}^{2+} \cdot{ }^{45,46} \mathrm{Chen}$ et $\mathrm{a}^{45}$ has utilized an NMDAR antagonist, D-2-amino-5-phosphonovalerate and a non-NMDAR antagonist, 6-cyano-7-nitroquinoxaline-2, 3-dione to protect neurons against glutamate-induced increase of $\mathrm{Ca}^{2+}$ and as a result, elevation of calpain and its cleavage product SBDPs have been shown to be attenuated.

Although different types of glutamate receptor antagonists, specifically those for NMDARs, have been successfully utilized in both in vitro and in vivo studies, they have failed categorically in clinical trials, because completely blocking NMDARs causes serious side effects, such as memory impairment, psychosis, and nausea. ${ }^{47}$ Thus, there is a growing effort to search for alternative ways of inhibiting glutamate excitotoxicity in order to minimize these side effects. Such an alternative strategy has been developed in an ischemic neuronal injury study. ${ }^{48}$ It has been previously reported that calpain-mediated cleavage of metabotropic glutamate receptor $1 \alpha(\mathrm{mGluR} 1 \alpha)$ had an important role in excitotoxicity, and a transactivating regulatory protein (TAT)-mGluR1 peptide, which was developed as an alternative to general receptor antagonists, was neuroprotective against this excitotoxicity. ${ }^{49}$ This peptide includes the calpain cleavage site of mGluR $1 \alpha$ and the peptide transduction domain of the TAT of human immunodeficiency virus (HIV). Since over-activation of glutamate receptors is also widely recognized as an initiating factor in ischemic neuronal death, Zhou et $\mathrm{al}^{48}$ tested this peptide in in vivo and in vitro neonatal hypoxia/ischemia
(H/I) models for its neuroprotective effect. Results have shown that the TAT-mGluR1 peptide is preventive against $\mathrm{H} / \mathrm{I}$-induced neuronal death in neonatal rats. The pathway of neuroprotection for the TAT-mGluR1 peptide is through blockade of calpain-mediated H/I-induced mGluR1 $\alpha$ degradation. However, calpain-mediated $\mathrm{H} / \mathrm{I}$-induced spectrin degradation is not affected by this peptide, suggesting that the neuroprotective effect of the peptide depends on inhibition of calpain-mediated mGluR1 $\alpha$ degradation, not on calpain inhibition. The results of this study indicated that TAT-mGluR1 peptide may be a strong alternative to prevent $\mathrm{H} / \mathrm{I}$-induced cytotoxic events, since it has the potential to cause milder side effects compared to those of glutamate receptor antagonists and calpain inhibitors.

One of the important features of NMDAR is its potential to form different subunit compositions that provide an advantage of having a number of receptor subtypes with different signaling and synaptic targeting characteristics. NMDARs are composed of an essential GluN1 subunit and different combinations of GluN2 (A-D) and GluN3 (A-B) subunits. In contrast with GluN1/GluN2 heteromers, which are well recognized by their high permeability to $\mathrm{Ca}^{2+}$, GluN3 subunits decrease NMDARs' $\mathrm{Ca}^{2+}$ permeability by at least tenfold. ${ }^{50,51}$ When this inhibitory GluN3 subunit is involved in the subunit composition, it causes both reduction in $\mathrm{Ca}^{2+}$ influx via NMDAR channels and alteration of synaptic targeting properties of the channel, indicating elimination of two neurodegenerative hallmarks of NMDARs. A recent study, utilizing the neuroprotective potential of GluN3A subunits against neurotoxin 3-nitropropionic acid (3-NP)-induced striatal excitotoxic damage in GluN3A over-expressing transgenic mice has demonstrated that mild over-expression of GluN3A protected striatal neurons from excitotoxic damage of 3-NP. ${ }^{52}$ Results have also shown that GluN3A-mediated neuroprotection is dose-dependent and potentially associated with inhibition of a $\mathrm{Ca}^{2+}$-dependent protease, calpain, which is evident from the decrease in 3-NP-induced cleavage of fodrin and Striatal-enriched protein tyrosine phosphatase (STEP) by calpain as compared to controls.

Attempts at treating neurodegenerative diseases utilizing NMDAR antagonists have far been unsatisfactory because it is difficult for most NMDARs' antagonists to efficiently cross the blood-brain barrier (BBB) to reach their target. ${ }^{53,54}$ Even if an inhibitor/antagonist can cross the BBB, direct inhibition of NMDARs has major drawbacks in that glutamate receptors assume a role in numerous vital cellular processes, and blockade of them may result in failure of these cellular processes. ${ }^{55-57}$ Thus, prevention of the downstream processes 
of excess glutamate receptor induction such as calpain overactivation, which drives neurons into apoptosis, may present a broader range of opportunities for neuroprotection. Calpain inhibition may provide advantages over glutamate receptor antagonists in that calpain is predominantly in its inactive proenzyme form under normal physiological conditions, and its significant activation occurs only under pathological conditions.

\section{Neuroprotection through inhibition of calpain activity or its downstream processes}

Although there is an intrinsic inhibitor of calpain named calpastatin, it has large active polypeptide fragments preventing it from crossing membrane barriers, which strongly reduces its therapeutic potential. ${ }^{58}$ By considering this problem, on the one hand, researchers have focused on designing exogenous small calpain inhibitor peptides, peptide-mimetics, or nonpeptidic analogs that have good cell permeability and low toxicity; on the other hand, researchers have been exploring novel strategies to overexpress or intrinsically up-regulate calpastatin. ${ }^{59-62}$

A number of reversible or irreversible exogenous calpain inhibitors such as AK275, ${ }^{63}$ MDL-28170, ${ }^{64}$ PD150606, ${ }^{62,65}$ SJA6017, ${ }^{66}$ A-705253, ${ }^{67,68}$ SNJ-1945, ${ }^{69}$ and Calpeptin ${ }^{70}$ are frequently used in experimental studies (Table 1). Researchers continually work on enhancing stability, specificity, potency, and efficiency of the synthetic calpain inhibitors by developing third and even fourth generation calpain inhibitors with improved pharmacological profiles. ${ }^{71-73}$

AK275 is one of the prototypic calpain inhibitors. In the very first exogenous calpain inhibitor experiments, AK275 was among the mostly preferred calpain inhibitors due to its selectivity, membrane permeability, solubility, and in vitro efficiency. Bartus et $\mathrm{al}^{63}$ tested AK275 for the first time for its neuroprotective effect on cortical ischemic damage-associated focal ischemia in rats. Over-activated calpain is one of the most potent participants of ischemic brain injury through different pathways such as hydrolysis of cytoskeletal and membrane proteins, ${ }^{74,75}$ and subsequent activation of certain enzymes like apoptotic caspase-3, which causes neuronal death. For this reason, these researchers used the middle cerebral artery occlusion method to induce focal ischemia, and they applied AK275 through supracortical perfusion. Results showed that abnormal calpain activity is an important factor for ischemia and that AK275 was a relatively reliable neuroprotectant for the treatment of focal brain ischemia.
In addition to AK275, calpeptin is another calpain inhibitor that may have an important function in protecting neurons against focal cerebral ischemia-reperfusion injury. Peng et $\mathrm{al}^{70}$ performed focal cerebral ischemia-reperfusion injury experiments in rat models to prove the neuroprotective effect of calpeptin through a potential mechanism of caspase-3 inhibition. The results showed that calpeptin reduced neuronal apoptosis in the hippocampal CA1 section of focal-cerebral ischemia-reperfusion-subjected rat brains by inhibiting caspase- 3 expression. This caspase- 3 inhibition may be due to inhibition of calpain, since it is well known that calpains can directly or indirectly modulate and activate p53, which in turn causes caspase- 3 activation. ${ }^{16}$ However, there is still need for further studies to reveal the exact mechanism of caspase- 3 inhibition by calpeptin.

MDL-28170 is another first generation calpain inhibitor which is membrane permeable and selective for calpain. It was used for the first time to prevent proteolysis of erythrocyte membrane-associated cytoskeletal proteins in rats. ${ }^{76}$ After that, Brorson et $\mathrm{al}^{64}$ performed experiments using MDL-28170 to prevent glutamate receptor-mediated neurotoxicity, which is known to cause $\mathrm{Ca}^{2+}$-dependent calpain over-activation. MDL-28170 was shown to successfully block proteolytic activity of calpain together with the advantage of limiting excitotoxicity even when applied 1 hour after calpain's toxic effects had been recorded.

In addition, MDL-28170 has also been shown to be neuroprotective against both neuronal apoptosis occurring as a result of oxidative stress and subsequent calpain activation through intracellular $\mathrm{Ca}^{2+}$-overload. ${ }^{77}$ In this study, using $\mathrm{H}_{2} \mathrm{O}_{2}$ to generate oxidative stress and using A23187 calcium ionophores to provide intracellular $\mathrm{Ca}^{2+}$ overload on neuron-like PC12 cells indicated significant increases both in calpain activity and mRNA expression of pro-apoptotic bax gene. However, pretreatment of PC12 cells with MDL-28170 has been shown to prevent both calpain activity and an increase in mRNA expression of proapoptotic bax gene, also suggesting a role for calpain in oxidative stress and $\mathrm{Ca}^{2+}$ influx-dependent neurodegeneration. ${ }^{77}$

A relatively less cytotoxic calpain inhibitor compared to other members of its class (such as MDL-28170, and calpain inhibitor I and II), named SJA6017, has been studied in diffuse traumatic brain injury mice models. ${ }^{66}$ This was the first use of a calpain inhibitor in a diffuse traumatic brain injury model, and results have shown that SJA6017, when administered early (20 minutes post-injury), provided an improvement on functional outcome after 24 hours of injury, indicating a possible therapeutic role for calpain inhibitors in traumatic brain injuries. 

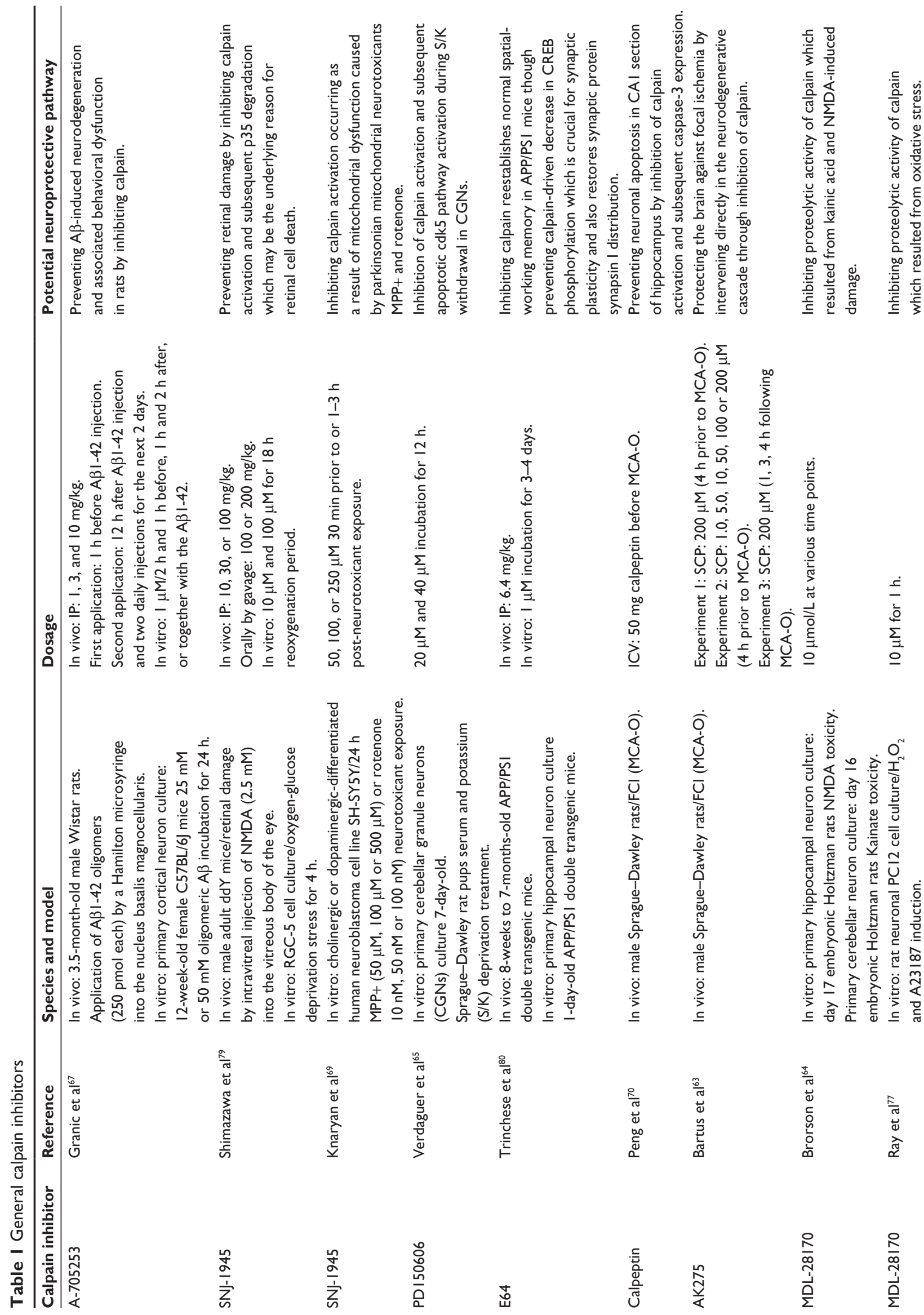

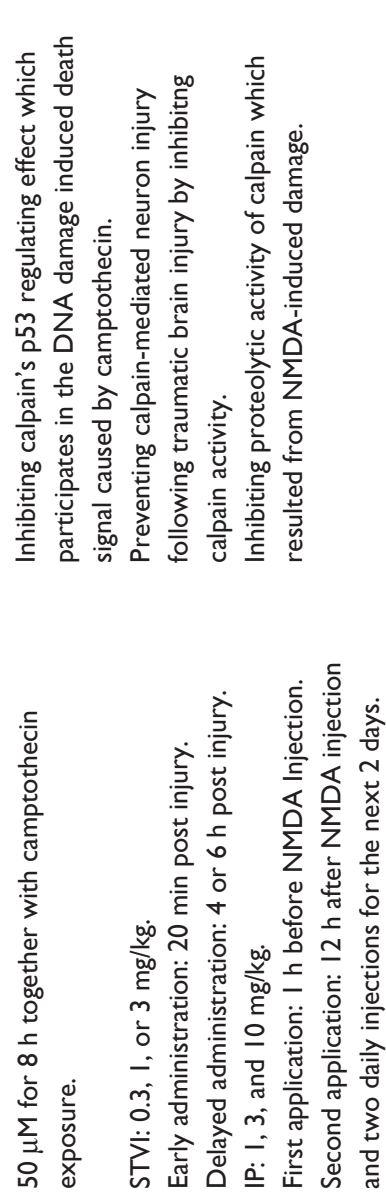

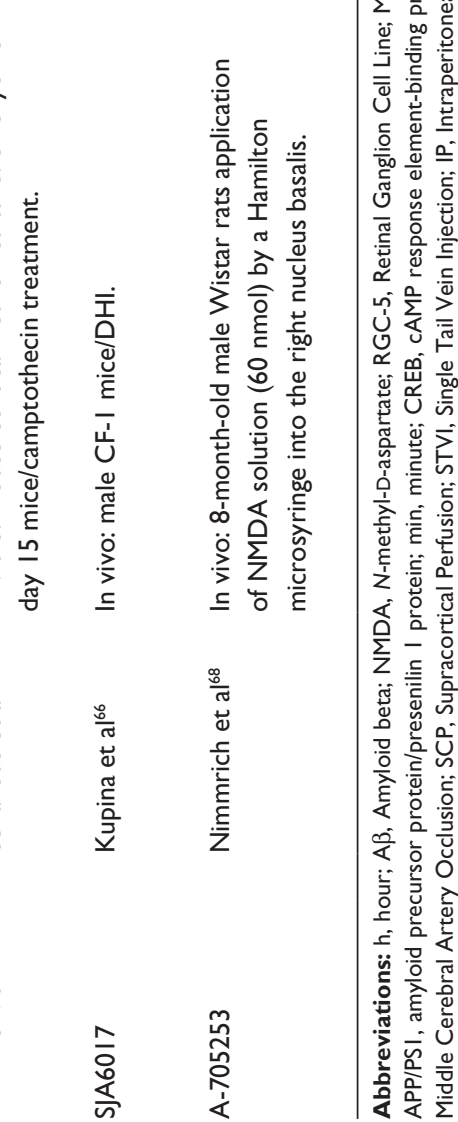

Since calpain is well characterized as a part of the NMDAR-mediated neurodegeneration, inhibition of calpain, instead of direct inhibition of NMDAR activity, is a rational way to prevent neurodegeneration in order not to interfere with NMDAR-mediated regulation of LTP and cognition. Thus, Nimmrich et al ${ }^{68}$ studied cholinergic neurons in disease-related animal models, which had excitotoxic lesions on cholinergic nucleus basalis magnocellularis of Meynert; these animal models also had a calpain inhibitor A-705253 to prevent excitotoxicity-induced neuronal decline without interfering with learning and memory processes. These researchers have shown that excitotoxicity seriously impeded novel object recognition ability, and that use of A-705253 calpain inhibitor prevented both this deficit and accompanying gliosis in a dose-dependent manner, while it did not inhibit LTP in hippocampal slices. Granic et al ${ }^{67}$ then tested A-705253 in a rat animal model of $A \beta$-induced neurodegeneration in which $A \beta$-induced lesions of nucleus basalis resulted in a significant decrease in cholinergic neuron number. A-705253 has been shown to prevent cholinergic neurodegeneration together with accompanied neuroinflammatory response, in a dose-dependent manner. ${ }^{67}$ From the results of this study, it can be concluded that calpain inhibition may also be a useful strategy to prevent $A \beta$-induced neurodegeneration.

On the other hand, in order to unravel the mechanisms of degeneration occurring in spinal cord neurons in the case of Parkinson's disease, SNJ-1945, a cell-permeable calpain inhibitor, has been tested for its neuroprotective activity in differentiated SH-SY5Y neuroblastoma cells that were exposed to two Parkinsonian neurotoxicants, the toxic cation 1-methyl-4-phenylpyridinium (MPP+) and rotenone to generate an experimental Parkinsonism model. $.^{58} \mathrm{MPP}+$ and rotenone, which have been previously shown to elevate the intracellular free $\mathrm{Ca}^{2+}$ levels and induce calpain over-activation in VSC 4.1 cells. ${ }^{64,78}$ The results of the study showed that SNJ-1945 pre-treatment significantly protected cells in terms of viability and cellular morphology against MPP+ and rotenone exposure, indicating that calpain inhibition is the true strategy to protect neurons against Parkinsonian neurotoxicants, and in this context, SNJ-1945 is a strong inhibitor of calpain. ${ }^{58}$

Neuroprotective effect of SNJ-1945 has also been evaluated to reduce retinal cell death caused by retinal cell damage. Researchers induced in vivo retinal damage in mice by injecting NMDA intravitreally, and then they administered SNJ-1945..$^{79}$ The in vitro part of the study was performed by inducing cell damage by using a 4-hour oxygen-glucose 
deprivation treatment followed by an 18-hour reoxygenation period in RGC-5 (a rat retinal ganglion cell line) cell culture. NMDA injection has been shown to cause calpain activation in vivo and in vitro by immunochemically measuring its cleaved products, spectrin and p35. The results of the study showed that SNJ-1945 significantly attenuated calpain activity, and reduced cell loss and also the number of positive cells for terminal deoxynucleotidyl transferase nick labeling in vivo and in vitro. ${ }^{79}$ These results not only indicate that calpain activation may be the key mechanism for retinal cell death, but also indicate that SNJ-1945 may be an effective neuroprotector in retinal diseases.

Despite their relatively efficient neuroprotective functions, there is a major limitation for the clinical use of available synthetic calpain inhibitors because of these inhibitors' lack of specificity towards calpains among other cysteine proteases and other proteolytic enzymes and related risk for these calpain inhibitors of inhibiting cysteine proteases other than calpains. Thus, developing alternative calpain inhibitors that have interaction with sites different from the catalytic site of calpain may provide enhanced specificity and potential for clinical use.

Alpha-mercaptoacrylate derivative PD150606 is one of these alternative kinds of calpain inhibitors, due to its relatively high specificity for calpains. It was described as a newly discovered class of calpain inhibitors by Wang and Yuen ${ }^{62}$ in 1994, owing to its feature of uncompetitive inhibition with respect to substrate. Since most calpain inhibitors are the peptides competing for the catalytic site of the protease, PD150606 differs from those inhibitors by its unique interaction with the calcium binding site of calpain rather than its catalytic site. The neuroprotective effect of PD150606 was shown for the first time by revealing its capability to inhibit hypoxic/hypoglycemic injury to cerebrocortical neurons and excitotoxic injury to Purkinje cells. ${ }^{63}$ Moreover, neuroprotection through PD150606 has also been tested in serum/potassium withdrawal-induced apoptosis of cerebellar granule cells. ${ }^{65}$ This research has demonstrated that $40 \mathrm{mM}$ PD150606 prevented serum/potassium withdrawal-mediated apoptosis by inhibiting calpain, indicating a therapeutic role for this type of calpain inhibitor.

Although synthetic calpain inhibitors (E64, BDA-410, etc) may also protect neurons in slow degenerative events such as Alzheimer's disease ${ }^{80,81}$ and in ischemic and traumatic injuries, the range of neurodegenerative conditions in which calpain inhibitors could provide benefits still remains uncertain. Most of the calpain inhibitor experiments up to now generally involved ischemic and traumatic injuries to the CNS, and there are a few studies showing the efficiency of calpain inhibitors in slow-progressing neurodegenerative diseases. ${ }^{82}$ Besides, calpain inhibitors, not having a complete specificity for calpain, can modulate other cysteine or serine proteases, which can have unknown effects on the progression of neurodegeneration. ${ }^{60}$ Thus, developing strategies for inhibiting downstream apoptotic processes of calpain overactivation rather than direct inhibition of calpain may be of great importance to eliminate all of the disadvantages of using calpain inhibitors.

Even though there have been many attempts to develop effective calpain inhibitors, none of the calpain inhibitors has been approved for clinical use. ${ }^{83}$ This is probably due to the low specificity of existing calpain inhibitors. Besides, combination of the nature and extent of enzyme inhibition is also important for effective inhibition of neuropathologic calpain activity. Thus, there is still a need for more efficient pharmacological approaches with reduced side effects.

Considering these negative aspects, in our laboratory, we have been searching for non-synthetic inhibitor candidates for calpain-mediated apoptosis in neurodegeneration. A novel and alternative cell cycle regulator protein Speedy/RINGO is quite remarkable in this respect, ${ }^{84}$ due to its attributed additional function in preventing caspase-dependent apoptosis in mitotic U2OS human osteosarcoma cells. ${ }^{85}$

As emphasized before, the calcium-activated proteolytic enzyme calpain is one of the key proteins that can directly or indirectly drive neurons into apoptosis. The indirect method is through cdk5, a non-mitotic kinase, which is up-regulated through calpain over-activation, a process followed by a subsequent increase in $\mathrm{p} 53$ and active caspase- 3 levels under neurodegenerative conditions. ${ }^{86-88}$ The direct method is the up-regulation of p53 by calpain itself, since it has been demonstrated that p53 can be modulated and activated by calpains. ${ }^{16}$ Speedy/RINGO is an atypical cell cycle regulator, synthesized only in mitotic cells, which has been shown to have protective effects in mitotic cells against apoptosis by inhibiting caspase- 3 activation in a p53-dependent manner. ${ }^{85,89}$ Our aim was to reveal possible protective effects of Speedy/RINGO against calpaininduced caspase- 3 activation in post mitotic neurons, which is crucial in terms of providing novel insights in preventing the caspase- 3 activation cascade in neurodegeneration. ${ }^{84}$ For this purpose, rat primary hippocampal neurons have been transfected with Speedy/RINGO expression construct, and then intrinsic calpain has been over-activated via the calcium ionophore. As a result, we have shown that calpain over-activation leads to the up-regulation of 
p53 and a subsequent increase in active caspase-3 levels in rat primary hippocampal neurons, indicating activation of apoptotic machinery in neurons. This calpain-directed caspase- 3 activation upon up-regulation of $\mathrm{p} 53$ is prevented by the expression of Speedy/RINGO in rat hippocampal neurons. Furthermore, 4',6-diamidino-2-phenylindole (DAPI) staining and terminal deoxynucleotidyl transferase dUTP nick end labeling assays have been performed for detection of apoptosis. Apoptotic analysis supported the finding that calpain over-activated neurons occurred in the early apoptotic stage, while calpain over-activated Speedy/ RINGO-expressing neurons were not apoptotic. ${ }^{84}$ Therefore, although its mechanism of action requires further elucidation, Speedy/RINGO may be a novel and alternative neuroprotectant, and it may act as a savior for neurons that are under apoptosis as a result of calpain activation due to caspase-3 activation.

On the other hand, there are efforts to up-regulate the intrinsic calpain inhibitor, calpastatin, because it may overcome the specificity, efficiency, water solubility, and BBB-crossing problems that synthetic, exogenous calpain inhibitors have. ${ }^{60,90}$ Taurine (2-aminoethanesulfonic acid), which is an organic acid widely distributed in mammalian tissues, has been utilized for this reason in an experimental study by Sun and $\mathrm{Xu} .{ }^{91}$ Taurine has a high cytoprotective potential due to its functions as a neurotransmitter, neuromodulator, modulator of intracellular calcium homeostasis, and anti-oxidant factor. ${ }^{92-94}$ Sun and $\mathrm{Xu}^{91}$ have investigated the neuroprotective role of intravenously administered taurine through affecting calpain/calpastatin actions in a rat model of focal cerebral ischemia. Results have indicated that taurine exerted dose-dependent neuroprotection by the mechanism of inhibiting the m-calpain- and caspase-3mediated apoptotic neuronal death via increasing calpastatin synthesis..$^{91}$ In order to use any substance as a neuroprotective agent, it is a prerequisite for that substance to have the ability to pass through the BBB. In this context, there is supporting evidence that taurine may pass through the $\mathrm{BBB}$, and hence, may be used as a neuroprotective agent in brain ischemia; for example, the demonstration of increased taurine levels in the brain after systemic administration of taurine indicates its ability to pass through the BBB. ${ }^{95,96}$ Identification of the carrier-mediated transport of taurine from vascular space to the brain ${ }^{97}$ and indication of linearly increased taurine levels in rat brains after intraperitoneal injection also support the idea that taurine can cross the BBB. ${ }^{98}$

In addition to up-regulating endogenous calpastatin, over-expressing it using expression vectors to inhibit calpain activity is also noteworthy. In a recent study, this technique has been utilized to inhibit calpain, which is suspected to be responsible for generation of toxic ataxin 3 fragments and hence pathogenesis of the Machado-Joseph disease, the most frequently found type of cerebellar ataxia. ${ }^{99}$ This study has produced adeno-associated viral vectors for overexpression of calpastatin and researchers have injected this viral construct into Machado-Joseph disease model rats. This study has demonstrated that calpain inhibition by over-expressed calpastatin significantly reduced the size and number of toxic ataxin 3 fragments and limited the progression of neurodegeneration in Machado-Joseph disease, pointing to another possible neuroprotective approach.

In contrast, calpastatin has been found to be markedly depleted in Alzheimer's disease brains of patients which in turn would cause uncontrolled activation of calpain. ${ }^{95}$ Based on this finding, Rao et a ${ }^{100}$ have produced human calpastatin over-expressing hCAST mice through an expression cassette containing Thy-1.1 promoter to investigate the effects of calpastatin in kainic acid-induced neurotoxicity, which has been shown to be closely associated with Alzheimer's disease. ${ }^{100}$ The results of this study have demonstrated that higher calpastatin levels inhibited kainic acid-induced cytoskeletal protein disruption, neurodegeneration, and accompanying reactive gliosis.

Although it is believed that calpain inhibition would have relatively less side effects, ${ }^{101,102}$ considering its inactive proenzyme form under normal physiological conditions, when necessary, upon high levels of calcium influx, calpain family members are activated and become crucially involved in multiple metabolic/regulatory pathways such as the cell cycle, differentiation, apoptosis, synaptic plasticity, and CNS development. ${ }^{5,9}$ Therefore, there still exist some limitations related to therapeutic use of calpain inhibitors, because multiple physiological functions of calpains might be disrupted by calpain inhibitors. Thus, approaches other than using synthetic calpain inhibitors, which may be unpractical and insufficient, may be strong alternatives for the treatment of neurological injuries and neurodegenerative disorders. In this context, using non-synthetic calpain-mediated apoptosis inhibitors such as Speedy/RINGO may be more realistic and beneficial in order not to interfere with the vital physiological functions of calpains in the cellular environment.

\section{Neuroprotection through estrogen receptors}

The female sex hormone estrogen has effects on the structure and function of the nervous system, such as supporting 
synaptic plasticity and preventing death of neurons, because brain regions related to sex differentiation have estrogen receptors. Together with this function, estrogen is also a neuroprotective anti-oxidant that may interact with neuroprotective signaling pathways in neurons. ${ }^{103}$

There is a growing body of evidence demonstrating that hormone replacement therapy, which is defined as using different types of estrogen alone or together with progestins, is very efficacious against a number of neurodegenerative conditions, such as Alzheimer's disease ${ }^{104,105}$ and Parkinson's disease. ${ }^{106,107}$

By taking into consideration this neuroprotective role of estrogen, researchers have been studying to find out if preventing calpain-mediated neurodegeneration via estrogen hormone and its receptors would be a convenient and powerful approach. For this purpose, Gamerdinger et al ${ }^{108}$ have analyzed estrogen and its receptors, $\mathrm{ER} \alpha$ and $\mathrm{ER} \beta$, with respect to their neuroprotective effect on over-expression of calpains under a massive attack of $\mathrm{Ca}^{2+}$ influx in ER $\alpha$ - and ER $\beta$-transfected human neuroblastoma SK-N-MC cells. The results of the study have revealed that calpain expression is inhibited by estrogen/ER $\alpha$ in a receptor subtype-specific fashion, since only ER $\alpha$ exerts this neuroprotective effect, not ER $\beta$. These data suggest an estrogen receptor subtypespecific neuroprotective action for calpain over-expressed degenerating neurons.

In addition, the neuroprotective role of estrogen has recently been tested specifically against amyloid toxicity by using an Alzheimer's disease rat model, which is produced by intra-hippocampal A $\beta 140$ injection. ${ }^{109} \mathrm{~A} \beta 140$ treatment has been shown to cause at least two times more calpain activation in the cerebral cortex, suggesting a role for calpains in amyloid toxicity. However, introduction of estrogen has been demonstrated to reduce $A \beta 140$ peptide levels in rat brain, and it has also been demonstrated that rats on estrogen therapy have not developed a behavioral dysfunction. Estrogen therapy has also been indicated to normalize A $\beta 140$-injected rats' proteolytic systems, both in the hippocampus and cortex regions. This effect of estrogen may be due to selective estrogen-dependent calpain synthesis, which has been indicated in a study about estrogen treatment of spinal cord injury; ${ }^{110}$ estrogen may cause reduction in calpain's enzymatic activity, even under massive $\mathrm{Ca}^{2+}$ influx, indicating an enhanced tolerance against $\mathrm{Ca}^{2+}$ toxicity.

Since estrogen is a steroid hormone that has effects on multiple metabolic pathways, it is not easy to develop a single estrogen-based approach for the treatment of calpainmediated neurodegeneration. However, it is still a strong therapeutic candidate as long as its mechanism of action is further analyzed.

\section{Conclusion}

Given the multifaceted nature of neurodegeneration, it does not seem to be easy to prevent onset or progression of neurodegeneration by clinically modulating a single process. However, while dissecting the underlying mechanisms of neurodegeneration, pathologic calpain activation has attracted great attention by many researchers for the reason that $\mathrm{Ca}^{2+}$-activated calpains have been shown to be activated and to trigger apoptotic machinery through different pathways in degenerating neurons. ${ }^{1-4}$ The main reason for abnormal calpain activation is known to be the massive $\mathrm{Ca}^{2+}$ influx that is frequently seen under neurodegenerative conditions. ${ }^{9,111,112}$ Glutamate neurotoxicity is one of the processes by which excessive $\mathrm{Ca}^{2+}$ influx is involved, and this process results in calpain over-activation, leading to neuronal apoptosis. ${ }^{37}$ Thus, many researchers have focused on both revealing the exact mechanism of glutamate receptor-related calpain activation, and on developing neuroprotective strategies in the context of glutamate receptor-related calpain activation. For this purpose, they have mainly performed experiments aimed at direct blockade of receptor activity to prevent excessive $\mathrm{Ca}^{2+}$ influx; they have used general receptor antagonists, ${ }^{45}$ alternative receptor blocking peptides, ${ }^{48}$ or over-expressing inhibitory subunits of the glutamate receptor, ${ }^{52}$ which reduces its activity. In contrast with its success in preventing calpain activation, direct blocking of glutamate receptors has too many drawbacks; direct blocking can result in disruption of cellular and metabolic integrity, because glutamate receptor activity is vital for regulating neuronal processes such as synaptic plasticity and memory function. ${ }^{55-57}$ From this point of view, rather than direct inhibition of glutamate receptor activity, studying of the inhibition of its downstream events, such as inhibiting calpain itself, sounds more realistic and beneficial if neuronal integrity is to be sustained. A number of synthetic peptide or non-peptide calpain inhibitors have been utilized for this purpose by different research groups. However, during these studies, many problems have arisen about specificity, efficiency, stability, and ability of these synthetic calpain inhibitors to cross the BBB. ${ }^{60}$

In order to overcome these problems, rather than complete inhibition of calpain, BBB-crossing competitive peptides or peptidomimetics, which inhibits active site of calpain or mimic certain substrates of calpain that are involved in neurodegenerative progress, can be designed to selectively prevent calpain's degradation activity on these substrates. ${ }^{113}$ 
In addition, for efficient and target-oriented delivery of these designed peptides to a desired location, a biocompatible delivery system can be used. This delivery system should be biocompatible, safe, and easy to manufacture. Examples of such delivery systems may be a fusion peptide, an antibody conjugate, an engineered virus, nanoparticles, or liposomes. ${ }^{114,115}$

On the other hand, apart from designing partial or complete calpain inhibitors, in order not to impede the essential functions of calpain, alternative approaches especially targeting downstream apoptotic events of calpain over-activation such as over-expression of anti-apoptotic Speedy/RINGO have successfully been tested in consideration of being beneficial against calpain-mediated apoptosis of neurons. ${ }^{84}$

Lastly, as a less studied approach, steroid hormone estrogen has been examined for preventing pathologic calpain activation in neurons considering its well-known neuroprotective properties. ${ }^{108}$ Results of these studies have shown that estrogen may be an effective neuroprotector against calpain-mediated neurodegeneration as long as some of its side effects are eliminated through a comprehensive understanding of its mechanism of action.

Taken together, studies reviewed here indicate the evergrowing picture of efficient, mechanism-based, targetoriented, feasible neuroprotective strategies against calpainbased neurodegeneration. Taking into consideration all the negative aspects of available methods, it is essential to change direction to more specific, efficient, practical, less harmful, and most importantly, target-oriented novel strategies to overcome apoptotic effects of calpain activation in neurons.

Last, but not the least, not only calpain-mediated neurodegeneration, but also other candidate underlying mechanisms of neuronal degeneration, point toward the development of a number of neuroprotective strategies that still require further analysis before they can be introduced into clinical practice. Although calpain over-activity is one of the most important and most potent underlying reasons for neurodegeneration, in order to remain realistic in our expectations, a multivisioned approach to developing therapeutic strategies for more than one degenerative mechanism is needed.

\section{Acknowledgments}

The "Speedy/RINGO over-expression to prevent calpainmediated apoptosis" study was funded by grants to Arzu Karabay from The Turkish Academy of Sciences Distinguished Young Scientist Award (TÜBA-GEBIP) and The Scientific and Technological Research Council of Turkey
(TÜBİTAK)-The Basic Sciences Research Group (TBAG) (grant number 108T811).

\section{Disclosure}

The authors declare no conflicts of interest in this work.

\section{References}

1. Liou AK, Zhou Z, Pei W, Lim TM, Yin XM, Chen J. BimEL upregulation potentiates AIF translocation and cell death in response to MPTP. Faseb J. 2005;19:1350-1352.

2. Araújo IM1, Verdasca MJ, Leal EC, et al. Early calpain-mediated proteolysis following AMPA receptor activation compromises neuronal survival in cultured hippocampal neurons. J Neurochem. 2004;91(6): 1322-1331.

3. Suzuki K, Hata S, Kawabata Y, Sorimachi H. Structure, activation, and biology of calpain. Diabetes. 2004;53(1):12-8.

4. Fukiage C, Azuma M, Nakamura Y, Tamada Y, Nakamura M, Shearer TR. SJA6017, a newly synthesized peptide aldehyde inhibitor of calpain: amelioration of cataract in cultured rat lenses. Biochim Biophys Acta. 1997;1361(3):304-312.

5. Yang L, Sugama S, Mischak RP. A novel systemically active caspase inhibitor attenuates the toxicities of MPTP, malonate, and 3NP in vivo. Neurobiology of Disease. 2004;17:250-259.

6. Neumar RW, Xu YA, Gada H, Guttmann RP, Siman R. Cross-talk between calpain and caspase proteolytic systems during neuronal apoptosis. J Biol Chem. 2003;278:14162-14167.

7. Moldoveanu T, Hosfield CM, Lim D, Elce JS, Jia Z, Davies PL. $\mathrm{A} \mathrm{Ca}(2+)$ switch aligns the active site of calpain. Cell. 2002;108(5): 649-660.

8. Reverter D, Sorimachi H, Bode W. The structure of calcium-free human m-calpain: implications for calcium activation and function. Trends Cardiovasc Med. 2001;11(6):222-229.

9. Polster BM, Basanez G, Etxebarria A, Hardwick JM, Nicholls DG. Calpain I induces cleavage and release of apoptosis-inducing factor from isolated mitochondria. J Biol Chem. 2005;280:6447-6454.

10. Hanna RA, Campbell RL, Davies PL. Calcium-bound structure of calpain and its mechanism of inhibition by calpastatin. Nature. 2008; 456:409-412.

11. Jánossy J, Ubezio P, Apáti A, Magócsi M, Tompa P, Friedrich P. Calpain as a multi-site regulator of cell cycle. Biochem Pharmacol. 2004;67(8):1513-1521.

12. Vanderklish P, Bednarski E, Lynch G. Translational suppression of calpain blocks long-term potentiation. Learn Mem. 1996;3: 209-217.

13. Sorimachi H, Ishiura S, Suzuki K. Structure and physiological function of calpains. Biochem J. 1997;328(3):721-732.

14. Lynch MA. Long-term potentiation and memory. Physiol Rev. 2004; 84(1):87-136.

15. Bednarski E, Vanderklish P, Gall C, Saido TC, Bahr BA, Lynch G. Translational suppression of calpain I reduces NMDA-induced spectrin proteolysis and pathophysiology in cultured hippocampal slices. Brain Res. 1995;694(1-2):147-157.

16. Sedarous M, Keramaris E, O'Hare M, et al. Calpains mediate p53 activation and neuronal death evoked by DNA damage. J Biol Chem. 2003;278:26031-26038

17. Touma E, Kato S, Fukui K, Koike T. Calpain-mediated cleavage of collapsin response mediator protein(CRMP)-2 during neurite degeneration in mice. Eur J Neurosci. 2007;26(12):3368-3381.

18. Raynaud F, Marcilhac A. Implication of calpain in neuronal apoptosis: A possible regulation of Alzheimer's Disease. FEBS Journal. 2006; 273:3437-3443.

19. Liu T, Perry G, Chan HW, Verdile G, et al. Amyloid-beta-induced toxicity of primary neurons is dependent upon differentiationassociated increases in tau and cyclin-dependent kinase 5 expression. J Neurochem. 2004;88:554-563. 
20. Raghupathi R. Cell death mechanisms following traumatic brain injury. Brain Pathol. 2004;14:215-222.

21. Shacka JJ, Roth KA. Regulation of neuronal cell death and neurodegeneration by members of the Bcl-2 family: therapeutic implications. Curr Drug Targets CNS Neurol Disord. 2005;4:25-39.

22. Gil-Parrado S, Fernandez-Montalvan A, Assfalg-Machleidt I, et al. Ionomycin-activated calpain triggers apoptosis: A probable role for Bcl-2 family members. J Biol Chem. 2002;277:27217-27226.

23. Putcha GV, Deshmukh M, Johnson EM Jr. BA fX translocation is a critical event in neuronal apoptosis: regulation by neuroprotectants, BCL-2, and caspases. J Neurosci. 1999;19:7476-7485.

24. Gao G, Dou QP. N-terminal cleavage of bax by calpain generates a potent proapoptotic $18-\mathrm{kDa}$ fragment that promotes bcl-2-independent cytochrome C release and apoptotic cell death. J Cell Biochem. 2000; 80:53-72.

25. O'Hare MJ, Kushwaha N, Zhang Y, et al. Differential roles of nuclear and cytoplasmic cyclin-dependent kinase 5 in apoptotic and excitotoxic neuronal death. J Neurosci. 2005;25:8954-8966.

26. Maccioni RB, Otth C, Concha II, Munoz JP. The protein kinase Cdk5: structural aspects, roles in neurogenesis and involvement in Alzheimer's pathology. Eur J Biochem. 2001;268:1518-1527.

27. Sharma AK, Rohrer B. Calcium-induced calpain mediates apoptosis via caspase-3 in a mouse photoreceptor cell line. J Biol Chem. 2004; 279(34):35564-35572.

28. Strachan GD, Koike MA, Siman R, Hall DJ, Jordan-Sciutto KL. E2F1 induces cell death, calpain activation, and MDMX degradation in a transcription independent manner implicating a novel role for E2F1 in neuronal loss in SIV encephalitis. J Cell Biochem. 2005;96: 728-740.

29. Pike BR, Flint J, Dutta S, Johnson E, Wang KK, Hayes RL. Accumulation of non-erythroid alpha II-spectrin and calpain-cleaved alpha II-spectrin breakdown products in cerebrospinal fluid after traumatic brain injury in rats. J Neurochem. 2001;78:1297-1306.

30. Saulle E, Gubellini P, Picconi B, et al. Neuronal vulnerability following inhibition of mitochondrial complex II: A possible ionic mechanism for Huntington's disease. Mol Cell Neurosci. 2004;25:9-20.

31. Smith MA, Casadesus G, Joseph JA, Perry G. Amyloid-beta and tau serve antioxidant functions in the aging and Alzheimer brain. Free Radic Biol Med. 2002;33:1194-1199.

32. Greenamyre JT, Porter RH. Anatomy and physiology of glutamate in the CNS. Neurology. 1994;44:7-13.

33. Dingledine R, Borges K, Bowie D, Traynelis SF. The glutamate receptor ion channels. Pharmacol Rev. 1999;51:7-61.

34. Rothman SM, Olney JW. Excitotoxicity and the NMDA receptor-still lethal after eight years. Trends Neurosci. 1995;18:57-58.

35. Lucas DR, Newhouse JP. The toxic effect of sodium L-glutamate on the inner layers of the retina. AMA Arch Ophthalmol. 1957;58: 193-201.

36. Lipton SA, Rosenberg PA. Excitatory amino acids as a final common pathway for neurologic disorders. N Engl J Med. 1994;330: 613-622.

37. Berliocchi L, Bano D, Nicotera P. $\mathrm{Ca}^{2}+$ signals and death programmes in neurons. Philos Trans R Soc Lond B Biol Sci. 2005;360: 2255-2258.

38. Lau A, Tymianski M. Glutamate receptors, neurotoxicity and neurodegeneration. Pflugers Arch. 2010;460:525-542.

39. Choi DW. Glutamate receptors and the induction of excitotoxic neuronal death. Prog Brain Res. 1994;100:47-51.

40. Miao Y, Dong L-D, Chen J, et al. Involvement of Calpain/p35-p25/ Cdk5/NMDAR Signaling Pathway in Glutamate-Induced Neurotoxicity in Cultured Rat Retinal Neurons. PLoS ONE. 2012;7(8):e42318. doi:10.1371/journal.pone. 0042318 .

41. Dhariwala FA, Rajadhyaksha MS. An unusual member of the Cdk family: Cdk5. Cell Mol Neurobiol. 2008;28(3):351-369.

42. Yip YP, Capriotti C, Drill E, Tsai LH, Yip JW. Cdk5 selectively affects the migration of different populations of neurons in the developing spinal cord. J Comp Neurol. 2007;503(2):297-307.
43. Fu X, Choi YK, Qu D, Yu Y, Cheung NS, Qi RZ. Identification of nuclear import mechanisms for the neuronal Cdk5 activator. $J$ Biol Chem. 2006;281(51):39014-39021.

44. Cruz JC, Tsai LH. Cdk5 deregulation in the pathogenesis of Alzheimer's disease. Trends Mol Med. 2004;10:452-458.

45. Chen J, Miao Y, Wang XH, Wang Z. Elevation of p-NR2AS1232 by $\mathrm{Cdk} 5 / \mathrm{p} 35$ contributes to retinal ganglion cell apoptosis in a rat experimental glaucoma model. Neurobiol Dis. 2011;43:455-464.

46. Wang J, Liu S, Fu Y, Wang JH, Lu Y. Cdk5 activation induces hippocampal CA1 cell death by directly phosphorylating NMDA receptors. Nat Neurosci. 2003;6:1039-1047.

47. Villmann C, Becker CM. On the hypes and falls in neuroprotection: targeting the NMDA receptor. Neuroscientist. 2007;13:594-615.

48. Zhou M, Xu W, Liao G, Bi X, Baudry M. Neuroprotection against neonatal hypoxia/ischemia-induced cerebral cell death by prevention of calpain-mediated mGluR $1 \alpha$ truncation. Experimental Neurology. 2009;218:75-82.

49. Xu J, Kurup P, Zhang Y, et al. Extrasynaptic NMDA receptors couple preferentially to excitotoxicity via calpain-mediated cleavage of STEP. J Neurosci. 2009;29:9330-9343.

50. Henson MA, Roberts AC, Perez-Otano I, Philpot BD. Influence of the NR3A subunit on NMDA receptor functions. Prog Neurobiol. 2010; 91:23-37.

51. Sasaki YF, Rothe T, Premkumar LS, et al. Characterization and comparison of the NR3A subunit of the NMDA receptor in recombinant systems and primary cortical neurons. J Neurophysiol. 2002;87: 2052-2063.

52. Turillas RM, Puerta E, Chowdhury D, et al. The NMDA receptor subunit GluN3A protects against 3-nitropropionic-induced striatal lesions via inhibiton of calpain activation. Neurobiology of Disease. 2012;48:290-298.

53. Milane A, Vautier S, Chacun $\mathrm{H}$, et al. Interactions between riluzole and ABCG2/BCRP transporter. Neurosci Lett. 2009;452:12-16.

54. Jinka TR, Rasley BT, Drew KL. Inhibition of NMDA-type glutamate receptors induces arousal from torpor in hibernating arctic ground squirrels (Urocitellus parryii). J Neurochem. 2012;122(5):934-940.

55. Kornhuber J, Weller M. Psychotogenicity and N-methyl-D-aspartate receptor antagonism: implications for neuroprotective pharmacotherapy. Biol Psychiatry. 1997;41(2):135-144.

56. Ratan RR, Murphy TH, Baraban JM. Oxidative stress induces apoptosis in embryonic cortical neurons. J Neurochem. 1994;62:376-379.

57. Sonkusare SK, Kaul CL, Ramarao P. Dementia of Alzheimer's disease and other neurodegenerative disorders - memantine, a new hope. Pharmacol Res. 2005;51:1-17.

58. Lee HL, Santé-Lhoutellier V, Vigouroux S, Briand Y, Briand M. Role of calpains in postmortem proteolysis in chicken muscle. Poult Sci. 2008;87(10):2126-2132.

59. Cuerrier D, Moldoveanu T, Campbell RL, et al. Development of calpain-specific inactivators by screening of positionalscanning epoxide libraries. J Biol Chem. 2007;282:9600-9611.

60. Carragher NO. Calpain inhibition: a therapeutic strategy targeting multiple disease states. Curr Pharm Des. 2006;12:615-638.

61. Zatz M, Starling A. Calpains and disease. N Engl J Med. 2005;352: 2413-2423.

62. Wang KKW, Yuen P. Calpain inhibition: an overview of its therapeutic potential. Trends Pharmacol Sci. 1994;15:412-419.

63. Bartus RT, Baker KL, Heiser AD, et al. Postischemic Administration of AK275, a Calpain Inhibitor, Provides Substantial Protection Against Focal Ischemic Brain Damage. J Cereb Blood Flow Metab. 1994;14:537-544.

64. Brorson JR, Marcuccilli CJ, Miller RJ. Delayed Antagonism of Calpain Reduces Excitotoxicity in Cultured Neurons. Stroke. 1995;26: 1259-1267.

65. Verdaguer E, Alvira D, Jimenez A, Rimbau V, Camins A, Palla's M. Inhibition of the cdk5/MEF2 pathway is involved in the antiapoptotic properties of calpain inhibitors in cerebellar neurons. British Journal of Pharmacology. 2005;145:1103-1111. 
66. Kupina NC, Nath R, Bernath EE. The novel calpain inhibitor SJA6017 improves functional outcome after delayed administration in a mouse model of diffuse brain injury. J Neurotrauma. 2001;18(11):1229-1240.

67. Granic I, Nyakas C, Luiten PGM, et al. Calpain inhibition prevents amyloid-b-induced neurodegeneration and associated behavioral dysfunction in rats. Neuropharmacology. 2010;59:334-342.

68. Nimmrich V, Szabo R, Nyakas C, et al. Inhibition of Calpain Prevents $N$-Methyl-D-aspartate-Induced Degeneration of the Nucleus Basalis and Associated Behavioral Dysfunction. J Pharmacol Exp Ther. 2008; 327(2):343-352.

69. Knaryan VH, Samantaray S, Park S, Azuma M, Inoue J, Banik NL. SNJ-1945, calpain inhibitor, protects SH-SY5Y cells against MPP+ and rotenone. J Neurochem. 2013;130(2):280-290.

70. Peng S, Kuang Z, Zhang Y, Xu H, Cheng Q. The protective effects and potential mechanism of Calpain inhibitor Calpeptin against focal cerebral ischemia-reperfusion injury in rats. Mol Biol Rep. 2011;38:905-912.

71. Lubisch W, Beckenbach E, Bopp S, et al. Benzoylalanine-derived ketoamides carrying vinylbenzyl amino residues: discovery of potent water-soluble calpain inhibitors with oral bioavailability. J Med Chem 2003;46:2404-2412.

72. Wang KK, Posner A, Raser KJ, et al. Alpha-mercaptoacrylic acid derivatives as novel selective calpain inhibitors. Adv Exp Med Biol. 1996;389:95-101.

73. Farkas B, Tantos A, Schlett K, Vilagi I, Friedrich P. Ischemia-induced increase in long-term potentiation is warded off by specific calpain inhibitor PD150606. Brain Res. 2004;1024:150-158.

74. Yamakawa H, Banno Y, Nakashima S, et al. Crucial role of calpain in hypoxic PC12 cell death: calpain, but not caspases, mediates degradation of cytoskeletal proteins and protein kinase C-alpha and -delta Neurol Res. 2001;23:522-530.

75. Wu H-Y, Yuen EY, Lu Y-F, et al. Regulation of N-Methyl-D-aspartate receptors by calpain in cortical neurons. J Biol Chem. 2005;280: 21588-21593.

76. Mehdi S, Angelastro MR, Wiseman JS, Bey P. Inhibition of the proteolysis of rat erythrocyte membrane proteins by a synthetic inhibitor of calpain. Biochem Biophys Res Commun. 1988;157:1117-1123.

77. Ray SK, Fidan M, Nowak MW, Wilford GG, Hogan EL, Banik NL. Oxidative stress and $\mathrm{Ca}^{2+}$ influx upregulate calpain and induce apoptosis in PC12 cells. Brain Res. 2000;852(2):326-334.

78. Samantaray S, Knaryan VH, Le Gal C, Ray SK, Banik NL. Calpain inhibition protected spinal cord motoneurons against 1-methyl-4-phenylpyridinium ion and rotenone. Neuroscience. 2011;192:263-274.

79. Shimazawa M, Suemori S, Inokuchi Y, et al. A Novel Calpain Inhibitor, ((1S)-1-((((1S)-1-Benzyl-3-cyclopropylamino-2,3-di-oxopropyl) amino)carbonyl)-3-methylbutyl)carbamic Acid 5-Methoxy-3oxapentyl Ester (SNJ-1945), Reduces Murine Retinal Cell Death In Vitro and In Vivo. JPET. 2010;332:380-387.

80. Trinchese $\mathrm{F}, \mathrm{Fa}$ ' M, Liu $\mathrm{S}$, et al. Inhibition of calpains improves memory and synaptic transmission in a mouse model of Alzheimer disease. J Clin Invest. 2008;118(8):2796-2807.

81. Battaglia F, Trinchese F, Liu S, Walter S, Nixon RA, Arancio O. Calpain inhibitors, a treatment for Alzheimer's disease: position paper. J Mol Neurosci. 2003;20(3):357-362.

82. Bartus RT, Dean RL, Cavanaugh K, Eveleth D, Carriero DL, Lynch G. Time-related neuronal changes following middle cerebral artery occlusion: implications for therapeutic intervention and the m'oie of calpain. J Cereb Blood Flow Metab. 1995;15,969-979.

83. Yuan J. Neuroprotective strategies targeting apoptotic and necrotic cell death for stroke. Apoptosis. 2009;14(4):469-477.

84. Yildiz-Unal A, Korulu S. Karabay A. Q111qc vSpeedy/RINGO Inhibits Calpain-Directed Apoptosis in Neurons. J Alzheimers Dis. 2012;31:779-791.

85. McAndrew CW, Gastwirt RF, Donoghue DJ. The atypical CDK activator Spy1 regulates the intrinsic DNA damage response and is dependent upon p53 to inhibit apoptosis. Cell Cycle. 2009;8:66-75.

86. Zhang J, Krishnamurthy PK, Johnson G. Cdk5 phosphorylates p53 and regulates its activity. J Neurochem. 2002;81:307-313.
87. Hamdane M, Bretteville A, Sambo AV, et al. p25/Cdk5-mediated retinoblastoma phosphorylation is an early event in neuronal cell death. J Cell Sci. 2005;118:1291-1298.

88. Alvira D, Ferrer I, Gutierrez-Cuesta J, Garcia-Castro B, Pallas M, Camins A. Activation of the calpain/cdk5/p25 pathway in the girus cinguli in Parkinson's disease. Parkinsonism Relat Disord. 2008;14: 309-313.

89. Gastwirt RF, Slavin DA, McAndrew CW, Donoghue DJ. Spy1 expression prevents normal, cellular responses to DNA damage: Inhibition of apoptosis and checkpoint activation. J Biol Chem. 2006;281: 35425-35435.

90. Higuchi M, Tomioka M, Takano J, et al. Distinct mechanistic roles of calpain and caspase activation in neurodegeneration as revealed in mice overexpressing their specific inhibitors. J Biol Chem. 2005;280:15229-37.

91. Sun M, Xu C. Neuroprotective Mechanism of Taurine due to Upregulating Calpastatin and Down-regulating Calpain and Caspase-3 during Focal Cerebral Ischemia. Cell Mol Neurobiol. 2008;28:593-611.

92. Schuller-Levis GB, Park E. Taurine and its chloramine: modulators of immunity. Neurochem Res. 2004;29:117-126.

93. Huxtable RJ. Physiological action of taurine. Physiol Rev. 1992;72: $101-163$.

94. Louzada PR, Lima AC, Mendonca-Silva DL, Noël F, De Mello FG, Ferreira ST. Taurine prevents the neurotoxicity of beta-amyloid and glutamate receptor agonists: activation of GABA receptors and possible implications for Alzheimer's disease and other neurological disorders. FASEB J. 2004;18:511-518.

95. Urquhart N, Perry TL, Hansen S, Kennedy J. Passage of taurine into adult mammalian brain. J Neurochem. 1974;22:871-872.

96. Pasantes-Morales H, Arzate ME. Effect of taurine on seizures induced by 4-aminopyridine. J Neurosci Res. 1981;6:465-474.

97. Tamai I, Senmaru M, Terasaki T, Tsuji A. $\mathrm{Na}^{+}$- and $\mathrm{Cl}^{-}$-dependent transport of taurine at the blood-brain barrier. Biochem Pharmacol. 1995;50:1783-1793.

98. Lallemand F, De Witte P. Taurine concentration in the brain and in the plasma following intraperitoneal injections. Amino Acids. 2004; 26:111-116.

99. Simões AT, Gonçalves N, Koeppen A, et al. Calpastatin-mediated inhibition of calpains in the mouse brain prevents mutant ataxin 3 proteolysis, nuclear localization and aggregation, relieving MachadoJoseph disease. Brain. 2012;135:2428-2439.

100. Rao MV, Mohan PS, Peterhoff CM, et al. Marked calpastatin (CAST) depletion in Alzheimer's disease accelerates cytoskeleton disruption and neurodegeneration: neuroprotection by CAST overexpression. J Neurosci. 2008;28(47):12241-12254.

101. Getz GS. Calpain inhibition as a potential treatment of Alzheimer's disease. Am J Pathol. 2012;181(2):388-391.

102. Saatman KE, Creed J, Raghupathi R. Calpain as a therapeutic target in traumatic brain injury. Neurotherapeutics. 2010;7(1):31-42.

103. Behl C. Estrogen as a neuroprotective hormone. Nat Rev Neurosci. 2002;3(6):433-442.

104. Kawas C, Resnick S, Morrison A, et al. A prospective study of estrogen replacement therapy and the risk of developing Alzheimer's disease: the Baltimore Longitudinal Study of Aging. Neurology. 1997;48(6): 1517-1521

105. Tang MX, Jacobs D, Stern Y, et al. Effect of oestrogen during menopause on risk and age at onset of Alzheimer's disease. The Lancet. 1996;348(9025):429-432.

106. Currie LJ, Harrison MB, Trugman JM, Bennett JP, Wooten GF. Postmenopausal estrogen use affects risk for Parkinson disease. Arch Neurol. 2004;61(6):886-888.

107. Marder K, Tang MX, Alfaro B, et al. Postmenopausal estrogen use and Parkinson's disease with and without dementia. Neurology. 1998; 50(4):1141-1143.

108. Gamerdinger M, Manthey D, Behl C. Estrogen receptor subtype-specific repression of calpain expression and calpain enzymatic activity in neuronal cells - implications for neuroprotection against $\mathrm{Ca}^{2+}$-mediated excitotoxicity. Journal of Neurochemistry. 2006;97:57-68. 
109. Lysenko LA, Kantserova NP, Rendakov NL, Sel'verova NB, Nemova NN. Calpain System Dysregulation in Rat Brain at BetaAmyloid Induced Neurodegeneration. Russian Journal of Bioorganic Chemistry. 2013;39(5):510-515.

110. Sribnick EA, Matzelle DD, Ray SK, Banik NL. Estrogen Treatment of Spinal Cord Injury Attenuates Calpain Activation and Apoptosis. J Neurosci Res. 2006;84:1064-1075.

111. Banay-Schwartz M, DeGuzman T, Palkovits M, Lajtha A. Calpain activity in adult and aged human brain regions. Neurochem Res. 1994; 19:563-567.

112. Sen A, Thom M, Martinian L, Jacobs T, Nikolic M, Sisodiya SM. Deregulation of cdk5 in hippocampal sclerosis. J Neuropathol Exp Neurol. 2006;65:55-66.
113. Li Q, Hanzlik RP, Weaver RF, Schönbrunn E. Molecular mode of action of a covalently inhibiting peptidomimetic on the human calpain protease core. Biochemistry. 2006;45(3):701-708.

114. Friedman AD, Claypool SE, Liu R. The smart targeting of nanoparticles. Curr Pharm Des. 2013;19(35):6315-6329.

115. Wang AZ, Gu F, Zhang L, et al. Biofunctionalized targeted nanoparticles for therapeutic applications. Expert Opin Biol Ther. 2008;8(8): 1063-1070.

\section{Publish your work in this journal}

Neuropsychiatric Disease and Treatment is an international, peerreviewed journal of clinical therapeutics and pharmacology focusing on concise rapid reporting of clinical or pre-clinical studies on a range of neuropsychiatric and neurological disorders. This journal is indexed on PubMed Central, the 'PsycINFO' database and CAS, and is the official journal of The International Neuropsychiatric Association (INA). The manuscript management system is completely online and includes a very quick and fair peer-review system, which is all easy to use. Visit http://www.dovepress.com/testimonials.php to read real quotes from published authors.

Submit your manuscript here: http://www.dovepress.com/neuropsychiatric-disease-and-treatment-journal 